Annals of Pure and Applied Mathematics

Vol. 12, No. 2, 2016, 211-220

ISSN: 2279-087X (P), 2279-0888(online)

Published on 6 December 2016

www.researchmathsci.org

DOI: http://dx.doi.org/10.22457/apam.v12n2a12

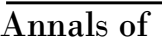

Pure and Applied

Mathematics

\title{
Size Multipartite Ramsey Numbers for Small Paths Versus Stripes
}

\author{
Chula Jayawardene and Lilanthi Samarasekara
}

Department of Mathematics, University of Colombo, Colombo 3, Sri Lanka

Corresponding author. Email: c jayawardene@yahoo.com

Received 30 October 2016; accepted 19 November 2016

Abstract. For graphs $G$ and $H$, the size balanced multipartite Ramsey number $m_{j}(G, H)$ is defined as the smallest positive integer $s$ such that any arbitrary two red/blue coloring of the graph $K_{j \times s}$ forces the appearance of a red $G$ or a blue $H$. In this paper we find the exact values of the multipartite Ramsey numbers $m_{j}\left(P_{3}, n K_{2}\right)$ and $m_{j}\left(P_{4}, n K_{2}\right)$.

Keywords: Ramsey theory, multipartite Ramsey numbers

AMS Mathematics Subject Classification (2010): 05C55, 05D10

1.Introduction

Let $G, H$ and $K$ be three finite graphs without loops and multiple edges. If for every two coloring (red and blue) of the edges of a complete graph $K_{n}$, there exists a copy of $H$ in the first color (red) or a copy of $K$ in the second color (blue), we say $G \rightarrow(H, K)$. The Ramsey number $r(G, H)$ is defined as the smallest positive integer $n$ such that $K_{n} \rightarrow(G, H)$. The classical Ramsey number $r(n, m)$ is defined as $r\left(K_{n}, K_{m}\right)$. Researches are now trying to approach this problem by using new techniques like fuzzy logic, genetic algorithms to improve the lower bound (see [7,8,9]). Another form of Ramsey numbers that originate when the complete graph is replaced by the complete bipartite graph in the above definition is called bipartite Ramsey numbers. More precisely, the bipartite Ramsey number $R_{b}(G, H)$ is defined as the smallest positive integer $n$ such that $K_{n} \rightarrow(G, H)$. The bipartite Ramsey number $R_{b}(n, m)$ is defined as $r\left(K_{n, n}, K_{m, m}\right)$. These bipartite Ramsey numbers have been explored extensively in the last decade (see $[2,3,4,5,6]$ ). One possible generalization of bipartite Ramsey numbers are the size multipartite Ramsey number $m_{j}(G, H)$ defined as the smallest positive integer $s$ such that $K_{j \times s} \rightarrow(G, H)$ where $K_{j \times s}$ represent the complete multipartite graph consisting of $j$ partite sets having exactly $s$ vertices in each 
Chula Jayawardene and Lilanthi Samarasekara

partite set. These numbers were introduced Burger and Vuuren and Syafrizal et al. (i.e., $[1,10])$ in the last decade. However, not much papers have been published in this area except for paths and cycles versus some small classes of graphs. (see $[1,10])$.

\section{Notation}

The order of the graph $G=(V, E)$ is denoted by $|V|$ and the number of edges in the graph is denoted by $|E|$. The neighborhood of a vertex $v \in V$ is defined as the set of vertices adjacent to $v$ and is denoted by $\Gamma(v)$.

If $v \in V$, the vertex $v$ along with the vertices adjacent to $v$ is denoted by $\overline{\Gamma(v)}$. The degree of a vertex $v, d(v)$, is defined as the cardinality of $\Gamma(v)$, i.e. $d(v)=|\Gamma(v)|$. The maximum degree of a graph $G(V, E)$ denoted by $\Delta(G)$ is defined as $\max \{d(v) \mid v \in V\}$. A path of size $n$ is a graph $P_{n}$, with $V=\left\{a_{1}, a_{2}, \ldots, a_{n}\right\}$ and edge set $E=\left\{\left(a_{1}, a_{2}\right),\left(a_{2}, a_{3}\right), \ldots,\left(a_{n-1}, a_{n}\right)\right\}$. A cycle of size $n$ is a graph $C_{n}$, with $V=\left\{a_{1}, a_{2}, \ldots, a_{n}\right\}$ and edge set given by $E=\left\{\left(a_{1}, a_{2}\right),\left(a_{2}, a_{3}\right), \ldots,\left(a_{n}, a_{1}\right)\right\}$. Given a matching $M$ (set of independent edges) of a graph $G(V, E)$ of size $t$, let $V(M)$ denote the $2 t$ vertices adjacent to the edges of matching $M$ and let $V(M)^{c}$ denote the vertices outside this matching of size $|V(G)|-2 t$.

\section{Small paths versus stripes}

Theorem 1. $m_{j}\left(P_{3}, n K_{2}\right)=\left\lceil\frac{2 n}{j}\right\rceil$

Proof: This is a direct consequence of Corollary 1 (see [10]) since we know $m_{j}\left(P_{3}, C_{2 n}\right)=\left\lceil\frac{2 n}{j}\right\rceil$ implies $m_{j}\left(P_{3}, n K_{2}\right)=\left\lceil\frac{2 n}{j}\right\rceil$.

Theorem 2. $m_{j}\left(P_{4}, n K_{2}\right) \geq\left\lceil\frac{2 n+1}{j}\right\rceil$ if $n>1$.

Proof: Let $n>1$. Consider the red/blue coloring given by $K_{j \times s}=H_{R} \oplus H_{B}$, where $s=\left\lceil\frac{2 n+1}{j}\right\rceil-1$, generated by coloring all edges leaving a singleton vertex(say $v$ ) of $K_{j \times s}$ by red and all the other edges by blue. Then, the graph has no red $P_{4}$, and $s j-1=\left(\left\lceil\frac{2 n+1}{j}\right\rceil-1\right) j-1<2 n$. Also the vertex $v$ is not adjacent to any vertices in blue. Therefore, the graph contains no blue $n K_{2}$. Hence, $m_{j}\left(P_{4}, n K_{2}\right) \geq\left\lceil\frac{2 n+1}{j}\right\rceil$.

In proving the theorem given below we will use the following lemma which is a direct consequence of Bondy's Lemma. 
Size Multipartite Ramsey Numbers for Small Paths Versus Stripes

Lemma 3. Consider the coloring given by $K_{j \times s}=H_{R} \oplus H_{B}$, where $j \geq 4$.

a) If $W$ is a subset of $V\left(K_{j \times s}\right)$ such that $|W| \geq 2 s+2$ and $\Delta\left(G_{R}[W]\right) \leq 1$ then $G_{B}[W]$ (the induced subgraph of the blue graph $G_{B}$ generated by the vertices of $W$ ) has a Hamilton cycle.

b) If $Y$ is a subset of $V\left(K_{j \times s}\right)$ such that $|Y| \geq 2 s$ and $\Delta\left(G_{R}[Y]\right)<1$ then $G_{B}[Y]$ has a Hamilton cycle.

\section{Theorem 4.}

$$
m_{j}\left(P_{4}, n K_{2}\right)=\left\{\begin{array}{cc}
2 & \text { if } n=1 \text { and } j=3 \\
\left\lceil\frac{2 n+1}{j}\right\rceil & \text { otherwise }
\end{array}\right.
$$

Proof: Clearly, $m_{j}\left(P_{4}, K_{2}\right)=2$ for $j=3$ and $m_{j}\left(P_{4}, K_{2}\right)=1$ for $j>3$. Therefore, it suffices to show $m_{j}\left(P_{4}, n K_{2}\right) \leq\left\lceil\frac{2 n+1}{j}\right\rceil$ for $n \neq 1$. Consider any red $P_{4}$ free coloring given by $K_{j \times s}=H_{R} \oplus H_{B}$, where $s=\left\lceil\frac{2 n+1}{j}\right\rceil$. Then the connected components of the red graph $H_{R}$ will consist of isolated vertices, a three cycle or $K_{1, m}$ for some $m \geq 1$. Define $N=\left\{v \mid v\right.$ is highest degree vertex of a red $K_{1, m}$ where $m>1$ or $v$ is any one of the vertices of a red $C_{3}$.

Case 1: If $j=3$

Let $V\left(K_{3 \times s}\right)=\left\{v_{i, k} \mid i \in\{1,2, \ldots, s\}\right.$ and $\left.k \in\{1,2,3\}\right\}$ and for a fixed $j \in\{1,2,3\}$ let $V_{j}=\left\{v_{i, j} \mid i \in\{1,2, \ldots, s\}\right\}$ represent the $j^{\text {th }}$ partite set. Let the red induced graph and the blue induced graph generated by the set $\left\{v_{i, k} \mid i \in\{p, p+1, \ldots, p+l\}\right.$ and $k \in\{1,2,3\}\}$ be denoted respectively by $G_{R}[p, p+1, \ldots, p+l]$ and $G_{B}[p, p+1, \ldots, p+l]$. Define $N[p, p+1]$ as the set of vertices of $N$ having the red degree greater than or equal to two in the induced subgraph of $G_{R}[p, p+1]$.

Claim 1 (Case 1): Any $G_{R}[p, p+1]$ has a red $K_{1,4}$ or $G_{B}[p, p+1]$ has a blue $3 K_{2}$. Proof: $|N[p, p+1]| \leq 2$ as the red degree of any vertex in $N[p, p+1]$ is greater than or equal to 2 in $G_{R}[p, p+1]$. If $|N[p, p+1]|=0$ then $G_{R}[p, p+1]$ can have at most three disjoint edges and thus the blue induced graph will have a $3 K_{2}$. Thus we have only 
Chula Jayawardene and Lilanthi Samarasekara

to consider the possibility when $|N[p, p+1]| \in\{1,2\}$. Therefore, if $G_{R}[p, p+1]$ has no red $K_{1,4}$ without loss of generality the only remaining possibilities for the maximal red graphs (That is, there are no red graphs on $G_{R}[p, p+1]$ satisfying the required conditions, that could be obtained by adding red edges to any one of these graphs) are illustrated in the following diagram.
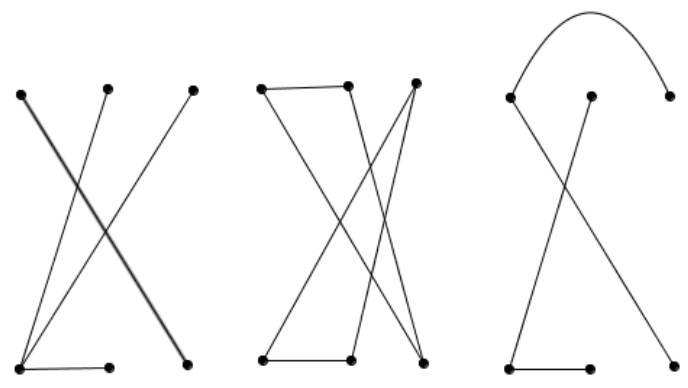

Figure 1: The three posibilities

But then its seen that in each of these possibilities the complement graph $\left(G_{B}[p, p+1]\right)$ will contain a $3 K_{2}$. Therefore, we can conclude that any $G_{R}[p, p+1]$ has a red $K_{1,4}$ or $G_{B}[p, p+1]$ has a blue $3 K_{2}$.

Claim 2 (Case 1): Any $G_{B}[p, p+1, p+2]$ has a blue $4 K_{2}$.

Proof: There are two possible cases.First suppose $G_{R}[p, p+1]$ has no red $K_{1,4}$. We know that $G_{B}[p+2]$ will contain a blue edge or $G_{R}[p+2]$ will contain a red $C_{3}$. In the first possibility we will get a blue $4 K_{2}$. In the later possibility suppose without loss of generality that $\left(v_{p, 1}, v_{p+1,2}\right)$ is a blue edge of the blue $3 K_{2}$ of the graph $G_{B}[p, p+1]$. Then in order to avoid a red $P_{4},\left(v_{p, 1}, v_{p+2,2}\right)$ and $\left(v_{p+1,2}, v_{p+2,1}\right)$ will have to be blue edges. Thus by replacing $\left(v_{p, 1}, v_{p+1,2}\right)$ by $\left(v_{p, 1}, v_{p+2,2}\right)$ and $\left(v_{p+1,2}, v_{p+2,1}\right)$ blue edges we will obtain a blue $4 K_{2}$.

Next suppose that $G_{R}[p, p+1]$ has a red $K_{1,4}$ with the root $v_{p, 1}$. Then independent of the structure of $G_{B}[p+2],\left(v_{p+2,1}, v_{p+1,3}\right),\left(v_{p, 3}, v_{p+2,2}\right)$, and $\left(v_{p+1,2}, v_{p+2,3}\right)$ will have to be blue edges. This would result in a blue $4 K_{2}$ as $\left(v_{p+1,1}, v_{p, 2}\right)$ is also a blue edge.

Claim 3 (Case 1): Any $G_{B}[p, p+1, p+2, p+3]$ has a blue $5 K_{2}$.

Proof: If one of $G_{R}[p, p+1]$ or $G_{R}[p+2, p+3]$ has no red $K_{1,4}$ clearly $G_{B}[p, p+1, p+2, p+3]$ will have a blue $5 K_{2}$ as required. Therefore, we may assume 
Size Multipartite Ramsey Numbers for Small Paths Versus Stripes

that $G_{R}[p, p+1]$ has a red $K_{1,4}$ and that $G_{R}[p+2, p+3]$ also has another red $K_{1,4}$. Without loss of generality the only possible red graphs are illustrated in the following diagram. However, eachof these two cases will result in a blue $5 K_{2}$.
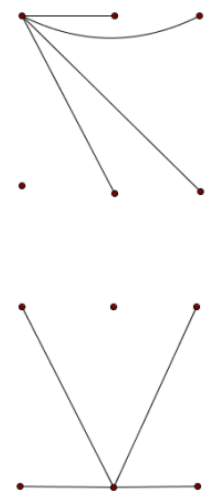
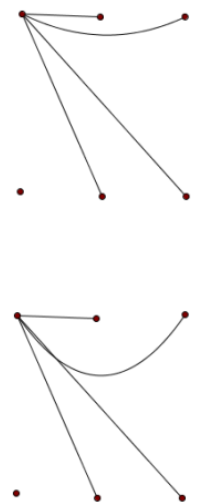

Figure 2: The four possibilities.

Claim 4 (Case 1): Suppose $l \geq 3$. Then any $G_{B}[1,2, \ldots, l]$ has a blue $m K_{2}$ where

$$
m= \begin{cases}\frac{3 l-1}{2} & \text { when } l \text { is odd } \\ \frac{3 l-2}{2} & \text { when } l \text { is even }\end{cases}
$$

Proof: We will use mathematical induction to prove this result. Suppose $l$ is a natural number such that $l \geq 3$. By claim 2 and claim 3, the result is true when $l=3$ and $l=4$ respectively. Letâ $\mathfrak{t M}^{\mathrm{TM}} \mathrm{S}$ assume that the result is true for all $p<l$.

When $l$ is odd, $G_{B}[1,2, \ldots, l-2]$ has a blue $m K_{2}$ where $m=\frac{3(l-2)-1}{2}$ (i.e. $m=\frac{3 l-7}{2}$ ). So, $G_{B}[1,2, \ldots, l-2]$ has one vertex not incident to the $m K_{2}$ (say $A$ ). By claim 1 , we have the following two situations.

Situation 1: $G_{B}[l-1, l]$ has a blue $3 K_{2}$.

Situation 2: $G_{R}[l-1, l]$ has a red $K_{1,4}$.

Under situation 1 , combining $G_{B}[1,2, \ldots, l-2]$ and $G_{B}[l-1, l]$ we obtain that $G_{B}[1,2, \ldots, l]$ has a blue $\left(\frac{3 l-7}{2}+3\right) K_{2}$ (i.e. a blue $\left(\frac{3 l-1}{2}\right) K_{2}$ ). 
Chula Jayawardene and Lilanthi Samarasekara

Under situation 2, the induced subgraph of $G_{B}[1,2, \hat{a}$ (say $H$ ) whose vertex set is $V\left(G_{B}[l-1, l]\right) \cup\{A\}$ has three blue edges that do not share end points. Therefore, combining the edges of the two matchings, we obtain that $G_{B}[1,2, \ldots, l]$ has a blue $\left(\frac{3 l-7}{2}+3\right) K_{2}$ (i.e. a blue $\left(\frac{3 l-1}{2}\right) K_{2}$ ).

When $l$ is even, $G_{B}[1,2, \ldots, l-2]$ has a blue $m K_{2}$ where $m=\frac{3(l-2)-2}{2}$ (i.e. $\left.m=\frac{3 l-8}{2}\right)$. So, $G_{B}[1,2, \ldots, l-2]$ has two vertices not incident to the $m K_{2}$. As argued above, by considering the two situations, we obtain that $G_{B}[1,2, \ldots, l]$ has a blue $\left(\frac{3 l-8}{2}+3\right) K_{2}$ (i.e. a blue $\left.\left(\frac{3 l-2}{2}\right) K_{2}\right)$. So, by mathematical induction, the result is true for all $l \geq 3$. Thus by the above claim, when $j=3$, any red $P_{4}$ free coloring given by $K_{j \times s}=H_{R} \oplus H_{B}$, where $s=\left\lceil\frac{2 n+1}{j}\right\rceil$ will have a blue $n K_{2}$.

Case 2: If $j \geq 4$

If $|N| \in\{0,1\}$ then at most one vertex in $N$ has at least two red edges incident to it. From this it is clear that when $|N| k\{0,1\}$, by Bondy's lemma, the theorem follows through. Thus, we will assume $|N| \geq 2$. Suppose that the the maximum degree of the red graph is $\Delta_{R}$ and achieved at $n_{1} \in N$. If $n \in K_{j \times s}$ then let $\Gamma_{R}(n)=\{v \mid(v, n)$ is an edge of $\left.H_{R}\right\}, \overline{\Gamma_{R}(n)}=\Gamma_{R}(n) \cup\{n\}$ and $\Gamma_{R}(N)=\left\{v \mid(v, m)\right.$ is an edge of $H_{R}$ for some $m \in N\}$. Define $N_{1}=N \backslash\left\{n_{1}\right\}$ (as $|N| \geq 2, N_{1} \neq \phi$ ).

Case 2.1: If $|N| \geq 2 s+1$

Since the vertices of $N$ that are not in the same partite set are connected to each other by blue edges the blue degree of any vertex of the induced subgraph on $N$ (denoted $\left.G_{B}[N]\right)$ is greater than or equal to $|N|-s \geq \frac{|N|}{2}$. Therefore, by Bondy's lemma $G_{B}[N]$ will contain a blue Hamilton cycle. By the definition of $N, G_{B}\left[N^{c}\right]$ will have at least $4 s+2$ vertices and again by Bondy's lemma(see Lemma 3) $G_{B}\left[N^{c}\right]$ will also contain a blue Hamilton cycle. Also as $|N|$ is greater than $2 s+1$ the two cycles will be connected by a blue edge. Thus $H_{B}$ will contain a Hamilton path. This will result in a blue $n K_{2}$ as required.

Case 2.2: If $\Delta_{R} \leq \frac{s j}{2}-s$ 
Size Multipartite Ramsey Numbers for Small Paths Versus Stripes

Then the blue degree of any vertex of the graph $H_{B}$ is greater than or equal to $s j-\left(\Delta_{R}+s\right) \geq \frac{s j}{2}$. Applying Bondy's Lemma to $H_{B}$ we get that it contains a blue Hamilton cycle. This will result in a blue $n K_{2}$ as required.

In each of the following two cases we apply Bondy's Lemma (see Lemma 3) to two sets separately. For each of these sets, we will require $j \geq 4$ condition to satisfy the degree condition of Bondy's Lemma.

Case 2.3: If $s+1 \leq|N| \leq 2 s$ and $\Delta_{R}>\frac{s j}{2}-s$

Then the number of vertices of $G\left[N_{1} \cup \Gamma_{R}\left(n_{1}\right)\right]$ is greater than $\frac{s j}{2}$. As we can assume that $|N| \geq 2$ there exist a vertex $v_{p+1} \in \overline{\Gamma_{R}\left(n_{1}\right)}$ and $n \in N_{1}$ such that $\left(n, v_{p+1}\right)$ is a blue edge. If $v_{p+1} \in \Gamma_{R}\left(n_{1}\right)$ let $x=v_{p+1}$. However if, $v_{p+1} \notin \Gamma_{R}\left(n_{1}\right)$ let $x$ be any element of $\Gamma_{R}\left(n_{1}\right)$. Applying Bondy's Lemma, separately to $G_{B}\left[N_{1} \cup\left(\Gamma_{R}\left(n_{1}\right) \backslash\{x\}\right)\right]$ (see Lemma 3 ) and $G_{B}\left[\left(N_{1} \cup \Gamma_{R}\left(n_{1}\right)\right)^{c} \cup\{x\}\right]$ (see Lemma 3), as $j \geq 5$ in this Case 2.3, we get that $G_{B}\left[N_{1} \cup\left(\Gamma_{R}\left(n_{1}\right) \backslash\{x\}\right)\right]$ and $G_{B}\left[\left(N_{1} \cup \Gamma_{R}\left(n_{1}\right)\right)^{c} \cup\{x\}\right]$ contain blue Hamilton cycles $n v_{2} v_{3} \ldots v_{p}$ and $v_{p+1} v_{p+2} \ldots, v_{s j}$ as indicated in the following diagram. Then we will obtain a blue Hamilton path in $H_{B}$ given by $v_{2} v_{3} \ldots v_{p} n v_{p+1} v_{p+2} \ldots v_{s j}$. This will result in a blue $n K_{2}$ as required.

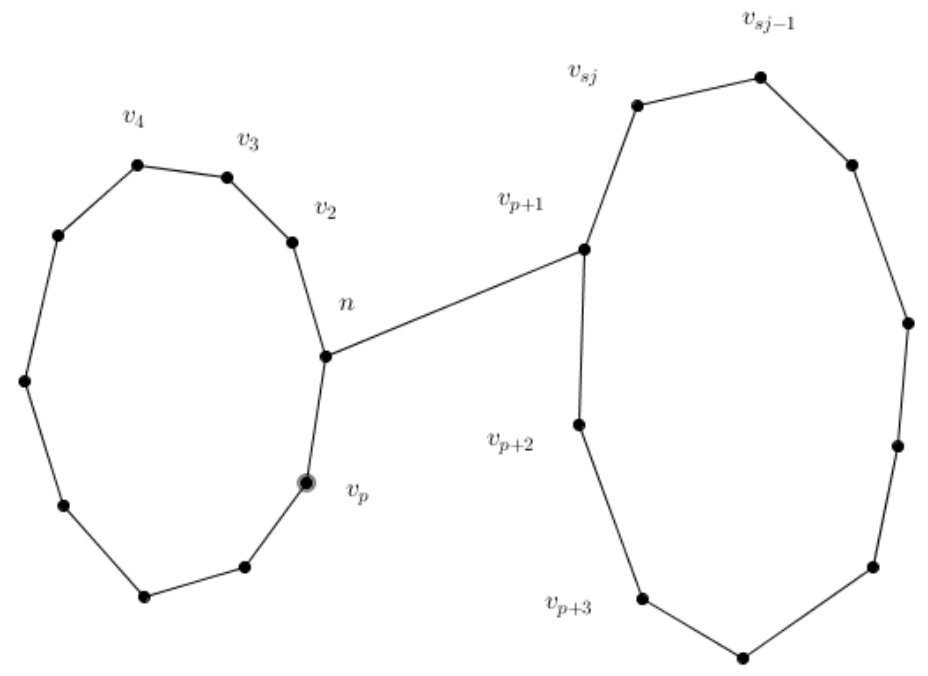

Figure 3: Case 2.3.

Case 2.4: If $|N| \leq s$ and $\Delta_{R}>\frac{s j}{2}-s$ 
Chula Jayawardene and Lilanthi Samarasekara

Claim 1 (Case 2.4): Suppose $N$ is not contained in any partite set. Then there is a matching of size $|N|-1$ where one edge of the matching is of the form $(n, m)$ where $n, m \in N$ and the other edges of the matching are between $N \backslash\{n, m\}$ and $\Gamma_{R}(N \backslash\{n, m\})$. Let $D$ denote the set of vertices incident to the blue matching of size $|N|-1$.

Remark 1. For any two distinct vertices $n$ and $m$ of $G_{R}[N]$ belonging to the same partite set, we can find a vertex in $\Gamma_{R}(m)$ adjacent to $n$ in blue and a vertex in $\Gamma_{R}(n)$ adjacent to $m$ in blue. Further, for any three distinct vertices $n, m$ and $k$ of $G_{R}[N]$ belonging to the same partite set, we can find a vertex in $\Gamma_{R}(m)$ adjacent to $n$ in blue, a vertex in $\Gamma_{R}(k)$ adjacent to $m$ in blue and a vertex in $\Gamma_{R}(n)$ adjacent to $k$ in blue.

Remark 2. For any three vertices $p, q$ and $r$ of $G_{R}[N]$ belonging to distinct partite sets, without loss of generality we can find a vertex in $\Gamma_{R}(p)$ adjacent to $q$ in blue and a vertex in $\Gamma_{R}(q)$ adjacent to $p$ in blue.

Remark 3. For any two vertices in $G_{R}[N]$ belonging to distinct partite sets by the definition of $N$ are connected by an blue edge.

Suppose $N$ is not contained in any partite set. Then, firstly by the repeated use of the first remark we can build a matching consisting of edges between $N$ and $\Gamma_{R}(N)$ such that if there are any remaining vertices then such vertices of $N$ will consist of singleton elements belonging to different partite sets. Secondly, by the repeated use of the second remark we can reduce the remaining elements of $N$ to consist of two elements of $N$ belonging to distinct partite sets or a singleton element of $N$.

If we were left with two vertices by the third remark we can increase the blue matching size by 1 as the two remaining vertices must be connected by a blue edge and obtain a matching of size $|N|-1$ as given in the claim. If we were left with one vertex we can switch one of the blue edges and obtain a similar matching as given in the claim. Then as $|D| \leq 2 s-2$, and $\left|D^{c}\right| \geq 2 s+2$, applying Bondy's Lemma (see Lemma 3) to $G_{B}\left[D^{c}\right]$ we get that it contains a blue Hamilton cycle.

This information is illustrated in Figure 4.

But then the blue matching of $G_{B}[D]$ and the blue matching of $G_{B}\left[D^{c}\right]$ together will give us the blue $n K_{2}$ as required.

Thus we are left with the case when $N$ is contained in some partite set $V_{i}$ of $K_{j \times s}$ and $j \geq 4$. Out of this first let us consider the case when $N$ is strictly contained in some partite set $V_{i}$ of $K_{j \times s}$ with $j \geq 4$ or if $N$ is equal to some partite set $V_{i}$ with $j \geq 5$. Then by remark 1 we will be able to find a blue matching of size $|N|$. Let $D_{1}$ denote the 
Size Multipartite Ramsey Numbers for Small Paths Versus Stripes

set of vertices incident to the blue matching of size $|N|$. But then, $\left|D_{1}^{c}\right| \geq 2 s+2$. Applying Bondy's Lemma (see Lemma 3) to $G_{B}\left[D_{1}^{c}\right]$ we get that it contains a blue Hamilton cycle. This information is illustrated in Figure 5..
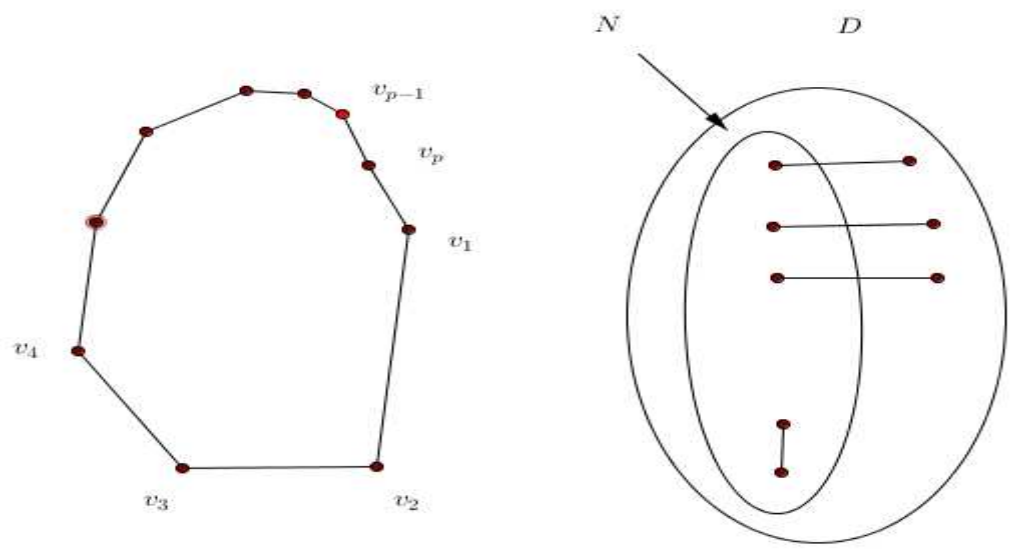

Figure 4: $G_{B}\left[D^{c}\right]$
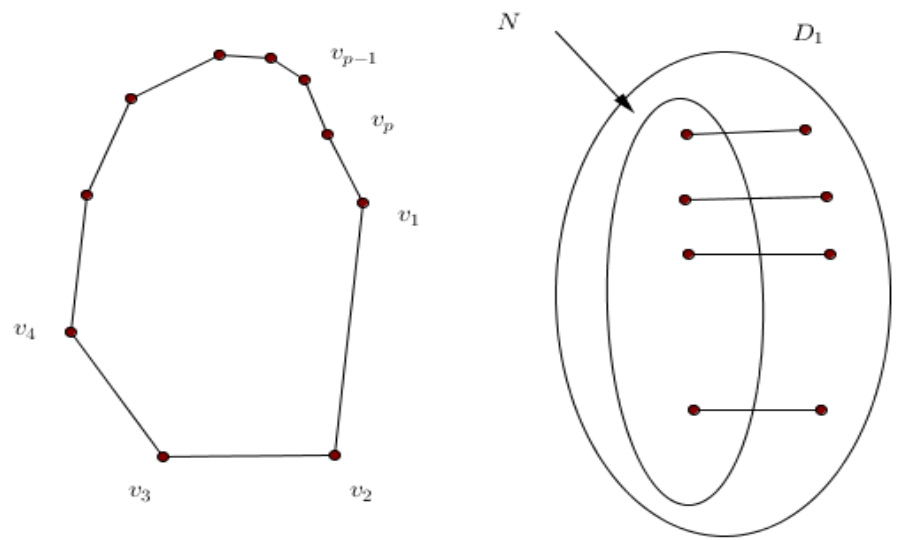

Figure 5: $G_{B}\left[D^{c}\right]$

Then the blue matching of $G_{B}\left[D_{1}\right]$ and the blue matching of $G_{B}\left[D_{1}^{c}\right]$ together will give us the blue $n K_{2}$ as required. Therefore, we are only left with the case where $N=V_{i}$ for some partite set $V_{i}$ with $j=4$. Then $\operatorname{deg}_{R}\left(n_{1}\right) \in\{s+1, s+2\}$ and either one of the following conditions will hold

a) $\operatorname{deg}_{R}\left(n_{1}\right)=s+2$ and the red degree of all the other vertices of $N$ is exacly 2 . 
Chula Jayawardene and Lilanthi Samarasekara

b) $\operatorname{deg}_{R}\left(n_{1}\right)=s+1$ and the red degree of all the other vertices of $N$ is exacly 2, and $H_{R}$ has one isolated vertex.

c) $\operatorname{deg}_{R}\left(n_{1}\right)=s+1$ and the red degree of all the other vertices of $N$ is exacly 2 except for one vertex of red degree 3 .

In each of the three cases as $\left|D_{1}^{c}\right|=2 s$. Then as $\Delta_{R}\left(G_{R}\left[D_{1}^{c}\right]\right)<1$ by applying Bondy's Lemma (see Lemma 3) to $G_{B}\left[D_{1}^{c}\right]$ we will get that it contains a blue Hamilton cycle, which will generate a blue $n K_{2}$ as required.

\section{REFERENCES}

1. A.P.Burger and J.H.van Vuuren, Ramsey numbers in complete balanced multipartite graphs. Part II: Size numbers, Discrete Math., 283 (2004) 45-49.

2. M.Christou, S.Iliopoulos and M.Miller, Bipartite Ramsey numbers involving stars, stripes and trees, Electronic Journal of Graph Theory and Applications, 1(2) (2013) 89-99.

3. R.J.Faudree and R.Schelp, Path-path ramsey-type numbers for the complete bipartite graph, Journal of Combinatorial Theory B, 19(2) (1975) 161-173.

4. V.Kavitha and R.Govindarajan, A study on Ramsey numbers and its bounds, Annals of Pure and Applied Mathematics, 8(2) (2014) 227-236.

5. S.P.Radziszowski, Small Ramsey numbers, Electronic Journal of Combinatorics, (rev 14) DS1 (2004).

6. C.C.Rousseau and J.Sheehan, On Ramsey numbers for books. Journal of Graph Theory, 2 (1978) 77-87.

7. Masulli, An introduction to fuzzy sets and systems, DISI - Dept. Computer and Information Sciences, University of Genova, Italy, (2010).

8. T.Pathinathan and J.Jesintha Rosline, Matrix representation of double layered fuzzy graph and its properties, Annals of Pure and Applied Mathematics, 8(2) (2014) 5158.

9. M.S.Sunitha and Sunil Mathew, Fuzzy graph theory: a survey, Annals of Pure and Applied Mathematics, 4(1) (2013) 92-110.

10. Syafrizal Sy and E.T.Baskoro, Lower bounds of the size multipartite Ramsey numbers, The 5th Mathematics, AIP Conf. Proc., 1450 (2012) 259-261. 